\title{
Uterine tamponade using condom catheter balloon in the management of non-traumatic postpartum hemorrhage
}

\author{
Renu Jain* \\ Department of Obstetrics and Gynecology, Gajra Raja Medical College, Gwalior, Madhya Pradesh, India \\ Received: 22 May 2018 \\ Accepted: 26 May 2018 \\ *Correspondence: \\ Dr. Renu Jain, \\ E-mail: renujain_1979@rediffmail.com \\ Copyright: (C) the author(s), publisher and licensee Medip Academy. This is an open-access article distributed under \\ the terms of the Creative Commons Attribution Non-Commercial License, which permits unrestricted non-commercial \\ use, distribution, and reproduction in any medium, provided the original work is properly cited.
}

\begin{abstract}
Background: In low-resource countries, Postpartum Hemorrhage (PPH) is the leading cause of maternal mortality. The intrauterine balloon tamponade was recently incorporated into the strategy to manage uterine atony. There are many types of tamponades. Among them, the condom catheter seems to be an efficient and economic intervention for the treatment of PPH in low-resource countries. The aim of present study was to evaluate the effectiveness of the condom catheter balloon in treating postpartum hemorrhage refractory to medical treatment.

Methods: The retrospective analysis of 22 cases of PPH, treated with a condom catheter as a conservative therapeutic option, was done in department of Obstetrics and Gynaecology, Gajra Raja Medical College, Gwalior, Madhya Pradesh, India.

Results: The condom catheter was successful in controlling PPH in $90.9 \%$ cases. It was effective in $94.44 \%$ women with uterine atony, who did not respond to uterotonic drugs. It was also effective in one case of placental implantation site bleeding and two cases of deranged coagulation function. In 2 cases it failed to control PPH and peripartum hysterectomy was done. The volume of saline solution used to fill the balloon was from $150-350 \mathrm{ml}$ and the time for which the balloon remained inflated was 36-48 hours. No case of febrile morbidity and wound sepsis was noted. There was no maternal death.

Conclusions: Its ease of use and high effectiveness make condom catheter a useful approach for the conservative management of PPH. This device reduces bleeding, shortens the hospital stay and avoids the need for more aggressive procedures.
\end{abstract}

Keywords: Maternal mortality, Postpartum hemorrhage (PPH), Peripartum hysterectomy

\section{INTRODUCTION}

Postpartum haemorrhage (PPH) is commonly defined as a blood loss of $500 \mathrm{ml}$ or more within 24 hours after birth. It affects approximately $2 \%$ of all women who give birth. It is associated not only with nearly one quarter of all maternal deaths globally but is also the leading cause of maternal mortality in most low-income countries. ${ }^{1}$

PPH is a significant contributor to severe maternal morbidity and long-term disability as well as to a number of other severe maternal conditions generally associated with more substantial blood loss, including shock and organ dysfunction. ${ }^{1}$

Postpartum hemorrhage describes an event rather than a diagnosis, and when encountered, its etiology must be determined. Common causes include bleeding from the placental implantation site, trauma to the genital tract and adjacent structures, or both. ${ }^{2}$ Unusual causes include placenta accreta, uterine inversion, and abnormalities of the haemostatic system. ${ }^{3}$ 
The most common cause of early PPH is uterine atony in $80-85 \%$ cases. In many women, uterine atony can at least be anticipated in advance of delivery. Although risk factors are well known, the ability to identify which individual woman will experience atony is limited. ${ }^{2}$

The diagnosis of PPH is obvious. First step in the management of uterine atony is the administration of uterotonic agents (oxytocin, methylergonovine, carboprost tromethamine and misoprostol). If uterine atony is unresponsive to fundal massage and the available oxytocic drugs, surgical techniques including uterine tamponade, uterine compression sutures, major vessel ligation and embolisation, and even hysterectomy will have to be considered. ${ }^{2}$

The invasive procedures involve laparotomy, require specialist expertise, may be associated with significant morbidity and may compromise future fertility. Interventional radiology offers a minimally invasive, fertility preserving alternative but requires special equipment, trained interventional radiologists and is not readily available in most obstetric units.

Uterine tamponade is a less invasive procedure which is simple, does not require major surgery, can be done within minutes, and will often immediately reduce or stop the bleeding. Thus, it may avoid the need for laparotomy and hysterectomy as well as need of blood transfusion with its inherent risk

Uterine tamponade requires developing intrauterine pressure to stop bleeding. This can be accomplished by insertion of a balloon that distends in the uterine cavity or by insertion of a uterine pack consisting of a gauze roll. ${ }^{3}$

At present WHO, RCOG and FIGO recommend the use of uterine balloon tamonade for refractory bleeding or if uterotonics are unavailable, after the exclusion of retained products and genital tract trauma. There are several reports in the literature describing success using hydrostatic balloon tamponade either alone or in combination with additional surgical methods. In such studies successful balloon tamponade outcomes have been reported in the range of $80-100 \% .{ }^{6}$ Different types of balloons have been used, including the SengstakenBlakemore tube, Rusch urological balloon, Bakri balloon, Foley catheters and condom catheters. ${ }^{6}$

Commercially available catheters like Bakri balloon has a prohibitory high cost, while condom catheter is a cheap and easily available alternative in low resource settings. So, the aim of this study was to evaluate the effectiveness of the uterine tamponade by condom catheter in treatment of postpartum hemorrhage.

\section{METHODS}

This retrospective study investigated the effectiveness of uterine tamponade by condom catheter in 22 cases of postpartum hemorrhage, refractory to medical treatment, at Department of Obstetrics and Gynaecology, Gajra Raja Medical College, Gwalior (M.P.).

All women with traumatic PPH and women with secondary $\mathrm{PPH}$ were excluded. Case records were reviewed and parameters were collected and analysed with regard to maternal age, parity, gestational age, laboratory test parameters, causes of $\mathrm{PPH}$, presence of risk factors, mode of delivery and indication of caesarean section. Variables related to the use of condom catheter and final outcome including need for additional procedures and associated complications were also analyzed.

Each of the delivery was conducted with active management of third stage of labor. A stepwise approach in the management of PPH was followed according to the hospital protocol and as soon as atonic PPH was diagnosed, uterine massage started and uterotonic agents, namely injection oxytocin, injection carboprost and tab misoprost per rectally were used in recommended doses for management of $\mathrm{PPH}$, with the supportive measures including intravenous fluids and blood or blood products before opting placement of intrauterine condom catheter. Maternal resuscitation was started while these procedures are being administered.

Condom catheter was prepared manually with all aseptic precautions by a technique described by Dr Nalini Mishra in her study. ${ }^{7}$

Simple things like a Foley's catheter of size 20-22, a packed condom, scissors, two $20-\mathrm{ml}$ syringes and 500-ml bottle of saline in a tray was required. Two rings of approximately 1-2 $\mathrm{mm}$ width were cut from the drainage tube of the catheter. The bulb of the catheter after inflating it with air was excised.

The condom was unfolded over distal one-third of the catheter. The rings were used to secure the condom over catheter leaving 1.5-2 cm from both the ends of condom. The tip of the Foley's catheter and condom together were excised to facilitate drainage of blood.

The condom catheter was inserted, vaginally in cases of vaginal delivery (using sponge holding forceps or manually into uterine cavity, using ring forceps to hold the cervix) or through the hysterotomy incision in cases of caesarean section.

Clinical success was defined as control of bleeding without further intervention. Analysis was done with the excel computer software and results were reported as percentage.

\section{RESULTS}

A total of 22 cases were identified in which condom catheter was used for uterine tamponade to control PPH. 
Table 1: Demographic characteristics.

\begin{tabular}{|c|c|c|}
\hline Characteristic & Number & Percentage \\
\hline \multicolumn{3}{|c|}{ Maternal age in years } \\
\hline$<19$ & 1 & 4.54 \\
\hline $20-24$ & 7 & 31.81 \\
\hline $25-29$ & 11 & 50 \\
\hline $30-35$ & 3 & 13.63 \\
\hline$>35$ & 0 & 0 \\
\hline \multicolumn{3}{|l|}{ Parity } \\
\hline Primigravidae & 8 & 36.36 \\
\hline Multigravidae & 14 & 63.63 \\
\hline \multicolumn{3}{|l|}{ Locality } \\
\hline Rural & 16 & 72.72 \\
\hline Urban & 6 & 27.27 \\
\hline \multicolumn{3}{|l|}{ Referral status } \\
\hline Direct & 8 & 36.36 \\
\hline Referred & 14 & 63.63 \\
\hline \multicolumn{3}{|l|}{ Booking status } \\
\hline Booked & 8 & 36.36 \\
\hline Unbooked & 14 & 63.63 \\
\hline
\end{tabular}

Table 1 demonstrates demographic characteristics of the patients. Majority of women were in age group 25-29 years $(50 \%)$, multigravidae $(63.63 \%)$ and from rural areas $(72.72 \%)$. Majority $(63.63 \%)$ of women were referred from different hospitals. $63.63 \%$ patients had no antenatal visits.

Table 2: Mode of delivery.

\begin{tabular}{|ll|l|}
\hline Mode of delivery & Number of cases & Percentage \\
\hline Vaginal delivery & 15 & 68.18 \\
\hline $\begin{array}{l}\text { Lower segment } \\
\text { caesarean section }\end{array}$ & 7 & 31.82 \\
\hline Total & 22 & 100.0 \\
\hline
\end{tabular}

Condom catheter was used to control bleeding, in 15 $(68.18 \%)$ cases delivered vaginally and in 7 (31.82\%) cases following Caesarean Section (Table 2).

Table 3: Indication of caesarean section.

\begin{tabular}{|ll|}
\hline Indication & No. of cases \\
\hline $\begin{array}{l}\text { Antepartum eclampsia with } \\
\text { unfavourable cervix }\end{array}$ & 1 \\
\hline Severe preeclampsia with IUGR & 1 \\
\hline $\begin{array}{l}\text { Twins with first fetus in breech } \\
\text { presentation }\end{array}$ & 2 \\
\hline $\begin{array}{l}\text { Placenta previa } \\
\text { Previous section with nonprogress }\end{array}$ & 2 \\
\hline Total & 1 \\
\hline
\end{tabular}

In 5 cases, cause of PPH following caesarean section was atonic uterus. Condom catheter was successful in controlling $\mathrm{PPH}$ in all 5 cases. In case of severe preeclampsia with IUGR, coagulation profile was deranged, so intrauterine catheter was placed simultaneously with transfusion of fresh frozen plasma and uterine bleeding was controlled. In case of placenta previa, condom catheter was successful in controlling placental implantation site bleeding (Table 3 ).

Table 4: Causes of PPH.

\begin{tabular}{|lll|}
\hline Cause & $\begin{array}{l}\text { Number } \\
\text { of cases }\end{array}$ & Percentage \\
\hline $\begin{array}{l}\text { Atonicity of uterus } \\
\text { Bleeding from placental }\end{array}$ & 18 & 81.81 \\
$\begin{array}{l}\text { implantation site in case } \\
\text { of placenta previa }\end{array}$ & 2 & 9.09 \\
\hline $\begin{array}{l}\text { Coagulation defect } \\
\text { Total }\end{array}$ & 2 & 9.09 \\
\hline
\end{tabular}

Condom catheter was used in $18(81.81 \%)$ cases of atonic $\mathrm{PPH}$ and $2(9.09 \%)$ cases of placental implantation site bleeding and coagulation defect each (Table 4).

Uterine tamponade by condom catheter was successful in all cases of PPH following vaginal delivery. All cases had atonic PPH following vaginal delivery except one. This case had jaundice with deranged coagulation profile. So intrauterine condom catheter was placed simultaneously with transfusion of fresh frozen plasma uterine, bleeding was controlled.

Table 5: The risk factors for atonic postpartum haemorrhage.

\begin{tabular}{|lll|}
\hline Risk factor & $\begin{array}{l}\text { Number of } \\
\text { cases }\end{array}$ & Percentage \\
\hline Grandmultiparity & 2 & 11.11 \\
\hline Severe Anemia & 4 & 22.22 \\
\hline Multiple pregnancy & 3 & 16.66 \\
\hline Prolonged labour & 4 & 22.22 \\
\hline Neonatal birth & 1 & 5.55 \\
weight $>$ 3.5 kg & 1 & 5.55 \\
\hline Polyhydramnios & 1 & 5.55 \\
\hline Eclampsia & 1 & 11.11 \\
\hline Not identified & 2 & 100 \\
\hline Total & 18 & \\
\hline
\end{tabular}

The main risk factors associated with atonic PPH were severe anemia and prolonged labour in $22.22 \%$ cases each, multiple pregnancy in $16.66 \%$ cases and grandmultiparity in $11.11 \%$ cases (Table 5).

According to our definition of effectiveness, as control of $\mathrm{PPH}$ not requiring any further intervention, uterine tamponade by condom catheter was effective in 20 $(90.9 \%)$ cases. Two women in whom the balloon failed to control PPH, were managed by peripartum hysterectomy.

After insertion, the balloon was inflated with normal saline until resistance was felt or bleeding was controlled. The volume of saline solution used to fill the balloon varied from $150-350 \mathrm{ml}$. Sterile roll gauze was used for 
vaginal packing to prevent condom catheter from falling out of uterus in all cases.

In cases of atonic PPH, to keep the uterus well contracted over the balloon, oxytocin infusion was continued for a minimum of 4 hours. The time for which the balloon remained inflated was from 36-48 hours. Antibiotics were administered for 5 days.

In present study, none of the patient developed febrile morbidity and wound sepsis following intrauterine condom catheter placement.

In present study there were no maternal deaths.

\section{DISCUSSION}

Postpartum haemorrhage is an obstetric emergency. It is the leading cause of maternal mortality in low income countries. Most deaths resulting from PPH occur during the first 24 hours after birth: the majority of these could be avoided through the use of prophylactic uterotonics during the third stage of labor and by timely and appropriate management. ${ }^{1}$

Recently, balloon tamponade has been widely used in the management of PPH unresponsive to standard medical management.

Currently, there are a number of proposed mechanisms by which the balloons are thought to exert their "tamponade" effect. The initial theory of the intrauterine balloon acting by exerting in inward-to-outward pressure "that is greater than the systemic arterial pressure" to prevent continual bleeding has been challenged. Studies of actual intrauterine pressures do not suggest that this is necessary, and alternative mechanisms of action have been proposed. These include: hydrostatic pressure effect on the uterine arteries, endometrial contact, vascular compression via myometrial stretch and myometrial activity secondary to myometrial stretching. Recently, cervical dilation resulting in myometrial activity has been demonstrated by electromyography. ${ }^{6}$

The idea of using a condom as a balloon tamponade was first generated and evaluated in Bangladesh, by Akhter S to fill a need and in response to the high cost of commercially available uterine balloon tamponade devices. $^{8}$

In a systemic review by Doumouchtsis SK et al, evaluating the success rate of different conservative methods of treatment of $\mathrm{PPH}$, the effectiveness of intrauterine balloon tamponade is similar to that of other methods, such as arterial embolization, surgical ligation of the uterine arteries or uterine compression suture. However, intrauterine balloon tamponade is less invasive, faster, and simple to perform; all of these features make this method a useful option as a first step in the conservative management of $\mathrm{PPH}^{9}$
In this retrospective study, we presented our centre's experience in treating $\mathrm{PPH}$ by condom balloon tamponade.

In present study, the condom catheter was successful in controlling PPH in $90.9 \%$ women. Dabelea et al, reported 23 cases with postpartum hemorrhage unresponsive to medical therapy but managed successfully with intrauterine balloon tamponade. ${ }^{10}$ Airede LR et al, reported four cases of PPH due to uterine atony in which intrauterine tamponade with inflated condom stopped bleeding. ${ }^{11}$

In present study, all women were treated with same device i.e. intrauterine condom balloon catheter. While in a study by Georgiou, different types of intrauterine balloons were used for the treatment of $\mathrm{PPH}$, with an overall success rate of $91.5 \% .{ }^{12}$ In study by Tirumuru S et al, Rusch balloon was used in 48 cases and Bakri balloon was used in 10 cases. ${ }^{13}$

In present study, condom catheter was used to control $\mathrm{PPH}$, in $15(68.18 \%)$ cases following vaginal delivery and in 7 (31.82 \%) cases following Caesarean Section. It was effective in all women with vaginal delivery and was not successful in two cases following caesarean section. Kadioglu BG et al, reported no significant difference between patients with vaginal delivery and caesarean section in terms of successful hemostasis rate. They reported the overall success rate of Bakri Ballooon alone to be as $84 \%$. $^{14}$

In present study, condom catheter was also effective in two cases of post partum hemorrhage due to deranged coagulation function, one was a patient with severe preeclampsia and other was a woman with jaundice. In a review by Georgiou $\mathrm{C}$, two cases using condom catheter were described in the management of PPH in women with impaired coagulation. ${ }^{12}$

In present study, it was also effective in controlling placental implantation site bleeding in a case of placenta previa. In a study by Alkis et al, balloon tamponade was effective in $87.5 \%$ cases of placenta previa. ${ }^{15}$ Its effect on bleeding from the placental bed, in cases of placenta previa, is the direct pressure of the balloon over the lower uterine segment.

Two cases of failure were observed. First was the case of atonic PPH, following caesarean section, done for previous section with non progress of labor, not managed by uterotonic drugs, so intrauterine condom catheter was placed. But bleeding was not controlled even after inflation of balloon with $500 \mathrm{ml}$ saline, so sub total hysterectomy was done. In another case of previous section with placenta previa, placenta found adhered to the lower uterine segment. On manual removal of placenta, placental bed started bleeding profusely. Firstly, intrauterine condom catheter was placed, but bleeding was not controlled and patient's vitals were started 
deteriorating, so total hysterectomy was performed to save the life of the mother. In both the cases, patients' post recovery period was uneventful and both were discharged healthy.

The main risk factors associated with atonic PPH were severe anemia, prolonged labour, multiple pregnancy and grandmultiparity. PPH may be aggravated by preexisting anemia and in such instances, the loss of a smaller volume of blood may still result in adverse clinical sequelae. The effectiveness of the condom balloon was not compromised by the presence of risk factors for PPH, a result that supports the use of this treatment in this type of patients.

The design of the balloon is an important consideration. In present study, condom catheter was assembled by the technique described by Mishra N. ${ }^{7}$ This condom catheter has a drainage port like commercially available uterinespecific devices like Bakri Balloon and thus helped in clinical assessment of the actual blood loss, an advantage that supports the use of this method for assembly of condom catheter.

In present study, $20 \mathrm{ml}$ syringe was used for inflation of condom balloon. Multiple authors used gravity inflation and an intravenous infusion set to inflate the condom, rather than using a syringe. ${ }^{16}$

In present study, vaginal pack was used in all cases, to prevent the condom catheter from falling out of uterus. In a systemic review by Tindell $\mathrm{K}$ et al, seven of the eight studies described using a vaginal pack (composed of sterile roller gauze). One study did not report any procedure to maintain placement of the uterine balloon tamponade. ${ }^{16}$

The volume of saline solution used to fill the balloon varied from $150-350 \mathrm{ml}$. However, the literature has provided examples of volumes ranging from $50-1500 \mathrm{ml}$ of normal saline that have been effective in controlling bleeding from an atonic uterus. ${ }^{6}$

The hypothetical risks of "over-distension" and "pressure" effects on the endometrium and myometrium secondary to filling are difficult to address as a term uterus can achieve a volume capacity that exceeds 400 ml. However, over-distension may have contributed to uterine rupture and subsequent hysterectomies in two cases of PPH in which balloon tamponade was used. ${ }^{17}$

In present study, in all 20 cases in which the condom catheter was successful, bleeding stopped within 5-20 minutes. In a systemic review by Tindell $\mathrm{K}$ et al, the time required for $\mathrm{PPH}$ to be controlled after placement of the condom catheter ranged from 4 to 15 minutes. ${ }^{16}$

In present study, the time for which the balloon remained inflated was 36-48 hours. Removal of the condom catheter began by emptying saline initially injected at a rate of $20 \mathrm{ml} / 15$ minutes. If bleeding resumed, the condom catheter could be reinflated and held in place for more hours. Otherwise, it was completely removed one hour later. Removal of the balloon was a one step procedure in all these women. Balloon deflation was done under very close observation preferably in the day time. There were no cases noted of re-bleeding after balloon removal. The length of time reported between insertion of the condom catheter and removal ranged widely, from 6 hours to as long as 72 hours. $^{6}$ In a study by Tirumuru $\mathrm{S}$ et al, the balloon was inflated for an average of 29 hours before removal (range $6 \mathrm{~h}$ to $80 \mathrm{~h}$ ). ${ }^{13}$

Side effects those likely related to the condom catheter are abdominal pain, fever, allergic reaction to latex and foul-smelling vaginal discharge. In present study, women only had lower abdominal pain of varying severity managed by oral or injectable analgesics.

In present study, none of the patients developed febrile morbidity and wound sepsis following condom catheter placement. Similarly, in study by Akhter $S$ et al, there was no intrauterine infection as documented by clinical signs and symptoms and culture and sensitivity of high vaginal swab. ${ }^{8}$ In a study by Tirumuru $S$ et al, there were 2 cases of endomyometritis. ${ }^{13}$

In present study, there were no maternal deaths. An important factor in the maternal mortality resulting from $\mathrm{PPH}$ is late recognition of the severity of the bleeding with a resulting delay in blood replacement. Unfortunately, when the woman with $\mathrm{PPH}$ decompensates, the opportunity for therapeutic intervention is limited and coagulopathy, renal failure, and death are difficult to avoid. ${ }^{3}$ In WHO systematic analysis of causes of maternal death, haemorrhage was the leading direct cause of maternal death worldwide, representing $27 \cdot 1 \%(19 \cdot 9-36 \cdot 2)$ of maternal deaths. More than two thirds of reported haemorrhage deaths were classified as postpartum haemorrhage. ${ }^{18}$

The simple technique to assemble the condom catheter and monitoring blood loss, along with its high effectiveness, make this a useful device for the conservative management of acute PPH that avoids the need for hysterectomy. As a result, the woman's fertility is conserved, blood loss is reduced, and the hospital stay is shortened. Nevertheless, randomized trials are needed to compare the effectiveness of balloon tamponade with other conservative modes of treatment, such as arterial embolization, surgical ligation of the uterine arteries or uterine compression suture.

\section{CONCLUSION}

Uterine preservation is an important issue when managing PPH. Condom catheter is effective in controlling non-traumatic $\mathrm{PPH}$ in $90.9 \%$ cases. It is simple, easy to use, easily available, with minimal adverse effects and cheap modality to manage non- 
traumatic PPH, especially in limited resource settings. It has a strong potential to save lives and reduce morbidity. But it is an improvised device that relies on the availability of the various components at the time of the PPH and the confidence of the health care provider in assembling and using the device.

Funding: No funding sources Conflict of interest: None declared

Ethical approval: Not required

\section{REFERENCES}

1. WHO. WHO recommendations for the prevention and treatment of post partum haemorrhage. Geneva: WHO; 2012. Available at http://www.who.int/reproductivehealth/publications/ maternal_perinatal_health/9789241548502/en/

2. Cunningham FG, Leveno KJ, Bloom SL, Hauth JC, Rouse DJ, Spong CY. Williams Obstetrics. 23rd edition. New York: McGraw Hill Medical; 2010:757-798.

3. Bhide A, Arulkumaran S, Damania KR, Daftary SN. Arias' Practical guide to high risk pregnancy and delivery. 3th edition. New Delhi: Elsevier;2008:348351 .

4. Royal College of Obstetricians and Gynaecologists. Prevention and management of post partum haemorrhage. Green-top Guideline No. 52. London: RCOG; 2009.

5. FIGO. FIGO Guidelines: Prevention and treatment of post partum hemorrhage in low resource settings. Int J Gynaecol Obstet. 2012;117:108-18.

6. Georgiou C. Current use of Balloon tamponade technology in the management of postpartum haemorrhage. Hypertens Res Pregnancy. 2014;2:110.

7. Mishra N, Agrawal S, Gulabani K, Shrivastava C. Use of an Innovative Condom Balloon Tamponade in Postpartum Haemorrhage: A Report. J Obstet Gynaecol India. 2016 Feb;66(1):63-7.

8. Akhter S, Begum MR, Kabir Z, Rashid M, Laila TR, et al. Use of a condom to control massive postpartum hemorrhage. Medscape General Med. 2003;5 (3):38.

9. Doumouchtsis SK, Papageorghiou AT, Arulkumaran S. Systematic review of conservative management of postpartum hemorrhage: what to do when medical treatment fails. Obstet Gynecol Surv. 2007;62:540-7.

10. Dabelea V, Schultze PM, Duffie RS Jr. Intra uterine balloon tamponade in the management of postpartum hemorrhage. Am J Perinatol. 2007; 24: 359-64.

11. Airede LR, Nnandi DC. The use of the condomcatheter for the treatment of postpartum hemorrhage. Trop Doct. 2008;38:84-6.

12. Georgiou C. Balloon tamponade in the management of postpartum haemorrhage: a review. BJOG. 2009;116:748-57.

13. Tirumuru S, Saba S, Morsi H, Muammar B. Intrauterine balloon tamponade in the management of severe postpartum hemorrhage: A case series from a busy UK district general hospital. Open J Obstet Gynecol. 2013;3:131-6.

14. Kadioglu BG, Tanriverdi EC, Aksoy AN. Balloon tamponade in the management of post partum hemorrhage: Three years of experience in a single center. Open J Obstet Gynaecol. 2016;6:698-704.

15. Alkis I, Karaman E, Han A, Ark CH, Buyukkaya B. The fertility sparing management of postpartum hemorrhage: A series of 47 cases of Bakri balloon tamponade. Taiwanese J Obstet Gynecol. 2015;54:232-5.

16. Tindell K, Garfinkel R, Abu-Haydar E, Ahn R, Burke T, Conn K, Eckardt M. Uterine balloon tamponade for the treatment of postpartum haemorrhage in resource-poor settings: a systematic review. BJOG. 2013;120:5-14.

17. Ajayi OA, Sant M, Ikhena S, Bako A. Uterine rupture complicating sequential curettage and Bakri balloon tamponade to control secondary PPH. BMJ Case Rep. 2013;2013.

18. Say L, Chou D, Gemmill A, Tunçalp O, Moller AB, et al. Global causes of maternal death: a WHO systematic analysis. Lancet Glob Health. 2014;2:323-33.

Cite this article as: Jain R. Uterine tamponade using condom catheter balloon in the management of nontraumatic postpartum hemorrhage. Int J Reprod Contracept Obstet Gynecol 2018;7:2617-22. 